\title{
Адаптация французского языка к социальным реалиям и потребностям граждан
}

(механизм обогащения)

\section{Этьен Кийо}

DOI: 10.30547/mediaalmanah.6.2020.188201

() Кийо Этьен

сотрудник Национального управления по продвижению французского языка и языков Франции, Министерство культуры Франции (г. Париж, Франция)
Языковая политика, которая проводится в настоящее время в целях адаптации французского языка к социальным реалиям и потребностям граждан, берет свое начало в 1960-1970 гг. Этот период, отмеченный быстрым ростом экономики, науки и техники, был также временем осознания необходимости выработки политики и создания структур, позволяющих приспособить французский язык к новым реалиям.

С одной стороны, французский язык, который к тому времени получил широкое распространение, усилил свои позиции: в частности, благодаря всеобщему доступу к среднему образованию, значительному росту числа студентов, а также вкладу радио и телевидения в унификацию речевой практики на всей территории страны.

С другой стороны, французский язык столкнулся, в научно-технической сфере, с необычайно быстрым появлением понятий, которые описывали инновации, новые технологические процессы, устройства, продукты и т.д. и по большей части имели обозначение на английском языке. Уже в 1962 г. Президент Республики генерал де Голль сетовал, в частности, «на избыточное употребление англосаксонской терминологии в военной области» и требовал исключать иностранные заимствования каждый раз, когда есть возможность 


\section{L'adaptation de la langue aux réalités sociales et aux besoins des citoyens}

(le dispositif d'enrichissement)

Étienne Quillot

(C) Quillot Étienne

représentant de la Mission Développement et enrichissement de la langue française

(Délégation Générale à la langue française et aux langues de France),

Ministère de la Culture de France

(Paris, France)

La politique linguistique qui est actuellement menée pour adapter la langue française aux réalités sociales et aux besoins des citoyens trouve ses origines dans le contexte des années 1960 / 1970. Cette période, qui fut marquée par un fort essor économique, scientifique et technique, fut également celle d'une prise de conscience de la nécessité de penser une politique et de se doter de structures permettant d'adapter la langue française aux réalités nouvelles.

D'un côté, la langue française, désormais largement partagée, était renforcée : notamment parce que l'accès à l'enseignement secondaire avait été généralisé, qu'il y avait une forte croissance du nombre d'étudiants, que la radio et la télévision contribuaient à unifier les pratiques langagières sur l'ensemble du territoire national.

De l'autre, la langue française était confrontée dans les secteurs scientifiques et techniques à l'apparition exponentielle de nouvelles notions qui décrivaient des innovations, de nouveaux procédés, de nouveaux appareils et produits, etc. Ces notions nouvelles arrivaient en France, pour la majorité, avec une désignation en anglais. Dès 1962, le Président de la République, le général de Gaulle déplora " notamment, dans le domaine militaire, l'emploi excessif de la terminologie anglo-saxonne " et demanda que soient proscrits les emprunts étrangers chaque fois que l'on pouvait employer un terme français, "c'est-à-dire dans tous les cas ".

Il y avait donc un risque de fractures, à la fois, avec un cloisonnement entre les catégories professionnelles qui utilisaient des jargons de plus en plus spécifiques, propres à leurs secteurs, et avec un cloisonnement entre la population et les représentants des secteurs professionnels dont les langages spécialisés s'écartaient de plus en plus de la langue générale, phénomène qui était accentué par le recours à des termes étrangers, c'est-à-dire anglais, alors que la maîtrise de cette langue par la population était encore très faible.

\section{Mise en place du dispositif d'enrichissement de la langue française}

Le dispositif d'enrichissement de la langue française est donc né dans ce contexte, au tournant des années 1970, en même temps que la France se dotait d'institutions et d'un cadre constitutionnel et légal lui permettant de mener une véritable politique linguistique en faveur du français : c'est-à-dire d'instaurer un « droit à la langue française ». 
употребить французский термин, «то есть во всех случаях».

Таким образом, существовал риск появления языковых барьеров с последующей языковой разобщенностью между профессиональными категориями, которые использовали все более и более специфический жаргон, принятый в этих областях, и языковой разобщенностью между населением и представителями профессиональных сообществ, специальная лексика которых все больше и больше отличалась от общеупотребительной. Причем это явление усиливалось за счет использования иностранных, а именно английских, терминов, в то время как владение этим языком среди населения оставалось на весьма низком уровне.

\section{Создание механизма обогащения французского языка}

Именно в таких условиях на рубеже 1970 гг. зародился механизм обогащения французского языка. Одновременно с этим во Франции были созданы институты, конституционная и законодательная база, позволявшая стране проводить действенную языковую политику в поддержку французского языка, то есть обеспечить «право на французский язык»:

- в 1966 г. создан Верховный комитет по защите и распространению французского языка (в 1989 г. на смену ему пришло Национальное управление по продвижению французского языка, а затем, в 2001 г., Национальное управление по продвижению французского языка и языков Франции);

- в 1975 и в 1994 гг. принято два закона об использовании французского языка - закон Ба-Лориоля от 31 декабря 1975 г. и закон Тубона от 4 августа 1994 г.;

- в 1992 г. в ст. 2 Конституции внесено положение о том, что «языком Республики является французский язык».

Поскольку обязанность использовать французский язык для юридических лиц публичного права и для лиц частного права при выполнении задач, связанных с государственной службой, была закреплена на законодательном уровне, то государство должно было проводить политику, направленную на обогащение французского языка новыми терминами, относящимися к сфере научно-технических инноваций и экономического развития.

Уже в начале 1970 гг. в различных министерствах были созданы комиссии экспертов. Учреждение двух таких комиссий заложило основы для создания соответствующей системы еще до принятия рамочного закона, который бы действовал для всех административных учреждений. Сначала 7 июля 1970 г. при Министерстве промышленного и научного развития была создана «комиссия по терминологии и неологии в области французской научной, технической и профессиональной лексики, относящейся к нефтяной отрасли». Затем 16 июня 1971 г. была создана комиссия по терминологии при Министерстве экономики и финансов. Таким образом, комиссии по разработке терминологии были созданы в отраслях, где заимствования из английского языка носили массовый характер. В их состав входили в основном специалисты соответствующих отраслей, а также лингвисты (специалисты в области терминологии, переводчики, редакторы).

Декрет № 72-19 от 7 января 1972 г. об обогащении французского языка был направлен на создание тематических комиссий по терминологии при всех центральных органах власти, во всех государственных секторах, то есть во всех министерствах. Перед комиссиями стояли две задачи: определить лакуны во французском словаре и предложить термины.

В декрет 1972 г. неоднократно вносились изменения, усиливавшие его содержание: в 1983, 1986 и в значительной степени в 1996 г., когда было добавлено положение о центральной комиссии по гармонизации работы (Главная комиссия по терминологии и неологии), и, наконец, в 2015 г., 
1966, création du Haut comité pour la défense et l'expansion de la langue française (en 1989 lui succédait la Délégation générale à la langue française, puis en 2001 la Délégation générale à la langue française et aux langues de France),

1975 et 1994, promulgation successive de deux lois relatives à l'emploi de la langue française, la loi « Bas-Lauriol » du 31 décembre 1975 et la loi « Toubon » du 4 août 1994,

1992, inscription à l'article de 2 de la Constitution que « la langue de la République est le français ".

L'obligation légale de l'usage du français s'imposant aux personnes morales de droit public et aux personnes de droit privé dans leur mission de service public, l'État a été conduit à mettre en œuvre une politique pour enrichir la langue française de nouveaux termes en relation avec les innovations scientifiques et techniques et le développement économique.

Dès 1970, des commissions composées d'experts ont été mises en place dans différents ministères. Les bases d'un réseau furent ainsi jetées avec la constitution de deux commissions, avant même la promulgation d'un texte cadre valable pour l'ensemble de l'administration. D'abord, le 7 juillet 1970, avec la création de la " commission de terminologie et de néologie du vocabulaire pétrolier français scientifique, technique et professionnel » auprès du ministre du développement industriel et scientifique. Ensuite, le 16 juin 1971, avec la création d'une commission de terminologie auprès du ministre de l'économie et des finances. Ces commissions chargées du développement de la terminologie furent donc créées dans des secteurs où les emprunts à l'anglais sont massifs; i elles étaient composées essentiellement de professionnels et de quelques linguistes (terminologues, traducteurs, rédacteurs...).

Le décret $n^{\circ} 72-19$ du 7 janvier 1972 relatif à l'enrichissement de la langue française visait à instituer des commissions de terminologie thématiques auprès de toute l'administration centrale - c'est-à-dire, dans tous les secteurs d'intervention de l'État (= dans tous les ministères). Les commissions avaient deux missions : identifier les lacunes du vocabulaire français et proposer des termes.

Le décret de 1972 a été adapté et renforcé à différentes reprises : en 1983, en 1986, notablement en 1996 avec l'ajout d'une commission centrale chargée de l'harmonisation des travaux (la Commission générale de terminologie et de néologie), et enfin en 2015, où cette commission a pris le nom de Commission d'enrichissement de la langue française. Chaque mise à jour du décret a permis d'améliorer l'organisation de l'ensemble du dispositif et d'associer un nombre croissant d'acteurs, issus en particulier des secteurs de la recherche et de l'enseignement supérieur.

Le réseau mis en place par décret a pour mission « de favoriser l'enrichissement de la langue française, de développer son utilisation, notamment dans la vie économique, les travaux scientifiques et les activités techniques et juridiques, d'améliorer sa diffusion en proposant des termes et expressions nouveaux pouvant servir de référence, de contribuer au rayonnement de la francophonie et de promouvoir le plurilinguisme » (décret n $96-602$ du 3 juillet 1996 relatif à l'enrichissement de la langue française, art. 1 - modifié par décret $n^{\circ}$ 2015-34l du 25 mars 2015, art. 3). 
когда эта комиссия была переименована в Комиссию по обогащению французского языка. Каждая актуализация декрета позволяла улучшить работу всего механизма и привлечь большее число участников, в особенности из таких сфер, как наука и высшее образование.

Задача сетевой системы, закрепленной декретом, заключается в том, чтобы «содействовать обогащению французского языка, развивать его использование, в частности в экономической жизни, научных работах, технической и юридической деятельности, усилить его распространение, предлагая новые термины и выражения, которые могли бы служить эталоном, способствовать расширению влияния франкофонии и продвигать многоязычие» (декрет № 96-602 от 3 июля 1996 г. об обогащении французского языка, ст. 1 - с изменениями, внесенными декретом № 2015-341 от 25 марта 2015 г., ст. 3).

Определения и термины, которые рекомендуются к употреблению путем опубликования в Официальном бюллетене и становятся обязательными для государственных ведомств, вначале изучаются специалистами и представителями научно-технических и лингвистических учреждений, гарантирующих как техническую точность, так и качество французского языка.

\section{Совершенствование механизма обогащения французского языка}

Работа по обогащению французского языка направлена на устранение языковых барьеров между профессиональными категориями, а также между специалистами и неспециалистами. Речь идет о том, чтобы поручить профессионалам какой-либо области составление точных и верных определений для того, чтобы ученые их не оспаривали и ими пользовались. При этом определения должны быть также понятными для людей, которые совершенно не разбираются в соответствующей области. При создании терминологических обозначений соблюдается тот же принцип вне зависимости от того, идет ли речь о понятиях, состоящих из слов общеупотребительного языка, или неологизмах.

Для выполнения этой задачи государство решило остаться в стороне и обратиться к тем, кто лучше всего разбирается в каждом из аспектов терминологической работы: специалистам, отвечающим за содержание (то есть за научно-технические объяснения), лингвистам, отвечающим за форму (то есть за редакционное качество) и представителям гражданского общества, выступающим в роли испытуемых (чтобы убедиться, что все граждане в состоянии понять предлагаемые термины). Для большей эффективности эта работа децентрализована и находится в ведении министерств и ведомств, которых напрямую касается соответствующая область, а государство ограничивается координацией и гармонизацией этого процесса, заказчиком которого оно и является.

Программа обогащения французского языка реализуется благодаря обширной сети групп экспертов по определенным тематикам (в различных министерствах), а также Комиссии по обогащению французского языка (находящейся в ведении премьер-министра) и различных партнеров. Группы экспертов (их в настоящий момент девятнадцать) занимаются терминологией в области инноваций в следующих отраслях (входящих в компетенцию министерств): сельское хозяйство и рыболовство; благоустройство, жилье и мобильность; автомобилестроение; биология; химия и материалы; культура и средства массовой информации; оборона; право и юстиция; экономика и финансы; обучение и высшее образование; атомная энергетика; ископаемое топливо; окружающая среда и устойчивое развитие; информатика; международные отношения; здравоохранение и социальные дела; наука и техника в области космоса; спорт; телекоммуникации. 
Les définitions et les termes proposés, qui sont recommandés par voie de publication au Journal officiel et s'imposent à l'administration, sont d'abord étudiés au cours d'une procédure associant plusieurs acteurs et institutions - techniques, scientifiques et linguistiques - garants tant de la précision technique que de la qualité de la langue française.

\section{Le réseau du dispositif d'enrichissement de la langue française}

L'enrichissement de la langue française a vocation à atténuer les fractures entre les catégories professionnelles et les fractures entre spécialistes et néophytes. Il s'agit par conséquent de confier à des professionnels d'un domaine la rédaction de définitions justes et précises pour que les scientifiques ne puissent les contester et se les approprient, tout en faisant en sorte que ces définitions soient aisément compréhensibles par des personnes ignorant tout du domaine. La création des termes respecte le même principe, qu'il s'agisse de termes composés de mots empruntés à la langue courante ou de néologismes.

Pour mener cette mission, l'État a choisi de rester en retrait et de faire appel aux plus compétents pour chacun des aspects du travail terminologique : les experts du domaine sont chargés du fond (c'est-à-dire des explications techniques et scientifiques), les linguistes de la forme (c'est-à-dire de la qualité rédactionnelle), des représentants de la société civile du « banc d'essai » (c'est-à-dire de s'assurer que tous les citoyens peuvent comprendre...). Pour une plus grande efficacité, la production terminologique est décentralisée, placée sous la responsabilité des administrations les plus directement concernées par le domaine, l'État se limitant à coordonner et à harmoniser la production terminologique dont il est le destinataire.

Le réseau du dispositif d'enrichissement de la langue française est constitué, principalement par des groupes d'experts thématiques (dans les différents ministères), ainsi que par la Commission d'enrichissement de la langue française (placée auprès du Premier Ministre) et par différents partenaires. Les groupes d'experts, actuellement au nombre de dix-neuf, sont chargés de la terminologie des innovations dans les domaines de compétence des ministères et ont pour intitulés : Agriculture et Pêche ; Aménagement, Habitat et Mobilités ; Automobile ; Biologie ; Chimie et Matériaux ; Culture et Médias ; Défense ; Droit et Justice ; Économie et Finances ; Éducation et Enseignement supérieur ; Énergie nucléaire ; Énergies fossiles ; Environnement et Développement durable ; Informatique ; Relations internationales; Santé et Affaires sociales ; Sciences et techniques spatiales; Sport ; Télécommunications.

D'autres groupes dit " ad hoc », constitués pour une durée limitée, traitent de thèmes précis, comme le vocabulaire de la cybersécurité et de la cyberdéfense, ou celui des nouveaux usages de l'hydrogène.

De nombreux partenaires viennent compléter le réseau :

- au premier rang desquels l'Académie française, dont l'avis conforme est requis pour publier au Journal officiel les termes retenus ;

- l'Académie des sciences ;

- des organismes de normalisation français, principalement l'Association française de normalisation ; 
Другие так называемые «специальные» группы, создаваемые на ограниченное время, занимаются точечными темами: например, лексикой по кибербезопасности и киберзащите, лексикой новых видов использования водорода.

Сетевую систему пополняют многочисленные партнеры:

- Французская академия (главный партнер), соответствующее заключение которой необходимо для публикации утвержденных терминов в Официальном бюллетене;

- Академия наук;

- учреждения стандартизации французского языка, главным образом Французская ассоциация по стандартизации;

- институты, отвечающие за лингвистическую политику и терминологию в других франкоговорящих странах, которые привлекаются к работе групп экспертов и с которыми систематически консультируются в ходе итогового рассмотрения терминов (главным образом Квебекское управление французского языка и Бюро перевода правительства Канады);

- Лингвистический департамент французского языка Главного управления переводов Европейской комиссии;

- университетские группы, которые оказывают лингвистическую поддержку специалистам в разных областях путем поиска возможных вариантов определения быстро развивающихся понятий;

- референтные субъекты для каждой области: органы государственной власти, институты, организации и профессиональные профсоюзы.

Сетевая система не функционирует горизонтально, как задумывалось в изначальной концепции декрета от 1972 г., когда звенья находились на одном уровне и конкурировали между собой. Не действует она и вертикально, как может показаться исходя из порядка прохождения этапов нынешней процедуры, когда одни субъекты (к примеру Комиссия по обогащению французского языка или Французская академия) могли бы иметь больший вес, чем другие. Скорее, она работает по образу планет или нейронов, каждый из которых играет свою роль, с множественными отношениями между звеньями и без жесткой иерархии. Такое децентрализованное функционирование является гарантией эффективности и нынешнего успеха сетевой системы.

\section{Формирование групп экспертов}

Задача групп экспертов состоит в том, чтобы для каждой отрасли привлечь всех заинтересованных субъектов к изучению и созданию терминов. Они состоят из квалифицированных специалистов и обязательных членов. Специалисты-эксперты это практически всегда добровольцы, за исключением нескольких человек, представляющих предприятие или университеты, которые вносят свой вклад в рамках исследовательских работ. Механизм обогащения французского языка не мог бы существовать без этих специалистов, которые составляют его основу, - около 300 человек (общее количество участников - 450).

Экспертов отбирают в зависимости от их компетенций, для наиболее широкого охвата всех аспектов соответствующей области. Например, в группу экспертов, отвечающую за терминологию в области здравоохранения, входят свыше двадцати специалистов, врачей и ученых во всех медицинских дисциплинах: анестезия и реанимация, онкология, хирургия, терапия, медицинская радиология, фармацевтика, психиатрия, ревматология и т.д., а также главы больниц и учреждений по уходу за пожилыми людьми и инвалидами и один специалист по медицинской информатике.

Состав групп экспертов дополняют обязательные члены. К примеру, для группы, отвечающей за здравоохранение, это будут руководитель Национального управления по продвижению французского языка и языков Франции или его представитель, представитель Французской академии из Словарной службы этого института. Кроме 
- des institutions chargées de la politique linguistique et de la terminologie dans d'autres pays francophones, qui sont associées aux travaux des groupes d'experts et consultées systématiquement lors de l'examen final des termes : principalement l'Office québécois de la langue française et le Bureau de la traduction du Gouvernement canadien ;

- le Département linguistique de langue française de la Direction générale de la traduction de la Commission européenne ;

- des équipes universitaires, qui apportent un soutien linguistique aux spécialistes des différents domaines en effectuant des recherches terminologiques sur les notions émergentes ;

- et des acteurs de référence pour chaque domaine : administrations, institutions, organisations et syndicats professionnels.

Le réseau ne fonctionne ni de façon horizontale, selon la conception initiale du décret de 1972, où les entités étaient côte à côte et en concurrence, ni de façon verticale, comme l'ordre des étapes de la procédure actuelle peut le laisser penser, où certains acteurs (par exemple la Commission d'enrichissement de la langue française ou l'Académie française) auraient plus de poids que d'autres, mais plutôt à l'image de planètes ou de neurones, chacun jouant son rôle, avec une multitude de relations entre toutes les entités et sans véritable hiérarchie. Ce fonctionnement décentralisé est la garantie de l'efficacité et du succès actuel de ce réseau.

\section{La composition des groupes d'experts}

Les groupes d'experts ont vocation à associer, pour chaque domaine, tous les acteurs concernés à l'étude et à la production des termes. Ils sont à la fois composés de personnalités, dites qualifiées, et de membres de droit.

Les personnalités - les experts - sont presque toujours bénévoles, à l'exception de quelques-uns, qui représentent une entreprise, ou d'universitaires qui apportent leur contribution dans le cadre de leurs travaux de recherche. Le dispositif d'enrichissement de la langue française ne pourrait exister sans ces professionnels qui constituent l'essentiel des effectifs, environ 300 personnes sur un total de 450 participants.

Ces experts sont sélectionnés en fonction de leurs compétences, en particulier dans le but de couvrir de la manière la plus large tous les aspects d'un domaine. Ainsi, par exemple dans le cas du groupe d'experts chargé de la terminologie de la santé, on trouve plus d'une vingtaine de spécialistes, médecins et chercheurs dans toutes les disciplines médicales : anesthésie et réanimation, cancérologie, chirurgie, médecine interne, médecine nucléaire, pharmacie, psychiatrie, rhumatologie, etc. ; mais aussi des directeurs d'hôpitaux et d'établissements de soins de personnes âgées ou handicapées, ou encore, un spécialiste d'informatique médicale.

Les membres de droit complètent la composition des groupes d'experts. Ils sont, par exemple pour le groupe chargé de la santé, le Délégué général à la langue française et aux langues de France ou son représentant, un représentant de l'Académie française, appartenant au Service du Dictionnaire de cette institution, ainsi que des représentants des principales institutions médicales et scientifiques: l'Académie des sciences, l'Association française de normalisation, l'Académie nationale de médecine, l'Académie nationale de pharmacie, l'Agence nationale 
того, речь идет о представителях основных медицинских и научных учреждений: Академии наук, Французской ассоциации по стандартизации, Национальной академии медицины, Национальной академии фармацевтики, Национального агентства по вопросам безопасности лекарственных препаратов и медицинских товаров, Высшего органа здравоохранения, независимой государственной организации, отвечающей за обеспечение качества обслуживания, - Национального института здравоохранения и медицинских исследований. И, наконец, представлены различные отраслевые управления Министерства здравоохранения.

Каждая группа экспертов формируется одинаковым образом с привлечением отраслевых специалистов и нескольких лингвистов, в основном представителей Национального управления по продвижению французского языка и языков Франции и Французской академии.

Также есть представители других групп сети, поскольку они поддерживают между собой тесные отношения для того, чтобы договариваться по общим терминам. Таким образом, эксперты, отвечающие за здравоохранение, работают рука об руку с биологами (к примеру, чтобы дать определение слову éligobiotique (элигобиотик) синтезированному антибиотику, образованному из оболочки бактериофага), химиками (фармацевтические термины) или специалистами в области окружающей среды (к примеру, чтобы дать определение и название на французском языке слову solastalgia, означающее «экотревогу, тревогу за окружающую среду»).

В каждом министерстве группы экспертов пользуются поддержкой высокопоставленного должностного лица, отвечающего за терминологию и французский язык. Последний инициирует и координирует в своем ведомстве действия по словарному обогащению французского языка. В его задачу входит создание групп экспертов в областях компетенции своего министерства, выявление специалистов, которые могут участвовать в работе, контроль за участием заинтересованных служб и органов, а также предоставление ресурсов, необходимых для эффективной работы групп. Высокопоставленное должностное лицо, по согласованию с экспертами, отвечает за распространение словаря среди специалистов. Наконец, к нему можно обратиться по любому вопросу использования французского языка в его министерстве - к примеру, относительно ясности и качества используемого администрацией языка по отношению к своей аудитории, феминизации названий профессий и должностей и т.д.

\section{Работа групп экспертов}

Первая задача экспертов заключается в обеспечении мониторинга неологизмов, главным образом посредством четырех категорий:

- новые понятия, которые они выявляют в своей профессиональной среде (предприятие, цех по разработке и производству, исследовательская лаборатория, университет и др.), в справочных, неспециализированных (см. журнал Nature Magazine) или отраслевых публикациях, на симпозиумах;

- понятия, относящиеся к специфическим темам, которые они изучают более глубоко (к примеру, термины, связанные с использованием электричества или водорода для движения транспортных средств);

- обращения от служб министерства (к примеру, необходимость отредактировать подготавливаемый подзаконный акт для того, чтобы точно определить термин или найти французский эквивалент);

• обращения от "широкой публики», которые по факту зачастую исходят от редакторов, специалистов по связям с общественностью, переводчиков, специалистов по терминологии, чиновников.

Вторая задача экспертов, которые собираются в среднем каждые два месяца, 
de sécurité du médicament et des produits de santé, la Haute autorité de santé qui est une organisation publique indépendante chargée de veiller à la qualité des soins -, l'Institut national de la santé et de la recherche médicale. Enfin, les différentes directions sectorielles du ministère de la santé sont représentées.

Chaque groupe d'experts est composé de manière similaire en faisant appel aux spécialistes du domaine, avec toujours une très large majorité de professionnels et quelques linguistes, principalement les représentants de la Délégation générale à la langue française et ceux de l'Académie française.

Il y a également des représentants d'autres groupes du réseau car ils entretiennent entre eux des relations étroites pour se concerter sur des termes communs. Ainsi les experts chargés de la santé travaillent main dans la main avec les biologistes (par exemple pour définir " éligobiotique », un antibiotique de synthèse formé de l'enveloppe d'un bactériophage), avec les chimistes (pour les termes de pharmacie) ou avec les spécialistes de l'environnement (par exemple pour définir et nommer en français solastalgia ou "éco-anxiété, anxiété due à l'environnement ").

Dans chaque ministère, les groupes d'experts bénéficient du soutien d'un haut fonctionnaire chargé de la terminologie et de la langue française. Celui-ci suscite et coordonne les actions d'enrichissement de la langue française dans son administration. Il est chargé de proposer la création de groupes d'experts dans les domaines de compétence de son ministère, d'identifier les professionnels susceptibles de participer aux travaux, de veiller à la participation des services et des organismes intéressés et d'apporter les ressources nécessaires au bon fonctionnement des groupes. Ce haut fonctionnaire est, en concertation avec les experts, responsable de la diffusion du vocabulaire auprès du public spécialisé.

\section{L'activité des groupes d'experts}

La première mission des experts est d'assurer la veille néologique, principalement à partir de quatre canaux :

les notions nouvelles qu'ils identifient dans leur milieu professionnel (entreprise, atelier de conception ou de fabrication, laboratoire de recherche, université...), les publications de référence, généralistes (cf. Nature Magazine) ou sectorielles, les colloques... ;

- les notions relatives à des thèmes spécifiques qu'ils étudient de façon plus systématique, par exemple les termes liés à l'utilisation de l'électricité ou de l'hydrogène pour la motorisation des véhicules... ;

- les demandes qui émanent des services de l'administration (par exemple, pour les besoins de rédaction d'un texte règlementaire en préparation, pour définir précisément un terme ou trouver un équivalent français) ;

- les demandes du " grand public », qui proviennent en fait souvent de rédacteurs, de communicants, de traducteurs, de terminologues, de fonctionnaires...

La seconde mission des experts, qui se réunissent en moyenne tous les deux mois, est de rédiger les définitions et de choisir le terme correspondant le plus approprié en passant par différentes étapes :

-en rassemblant toutes les informations et la documentation disponibles (contextes, occurrences, extraits issus de dictionnaires ou de bases de données...), avec l'aide des deux équipes universitaires de linguistes, 
заключается в составлении определений и выборе наиболее подходящего соответствующего термина после:

а) сбора всей доступной информации и документации (контекст, встречаемость слов, словарные статьи или базы данных и т.д.) при помощи двух университетских групп лингвистов;

б) одновременной консультации с партнерами по механизму (к примеру, с Квебекским управлением французского языка или Французской ассоциацией по стандартизации) с целью получения их заключения;

в) избирательного обращения к специалистам вне сети, в частности, когда изучаемое определение относится к аспекту данной области, в которой эксперты полностью не разбираются.

Затем в промежутке между заседаниями термин разбирается одним или несколькими докладчиками, которые отвечают за то, чтобы представить понятие другим экспертам, сформулировать предварительное определение и озвучить предложения по термину. Окончательная редакция определения, а также возможные примечания и выбор термина проходят на заседании. Этим занимаются эксперты, а лингвисты присутствуют лишь для консультации и передачи своих терминологических знаний экспертам. Таким образом, в ходе обсуждений и изучения терминов эксперты приобретают навыки терминологической экспертизы и становятся все более и более самостоятельными. После того как у группы экспертов появляется удачная серия терминов, она передает ее в Комиссию по обогащению французского языка.

\section{Комиссия по обогащению французского языка = площадка для тестирования}

Комиссия обеспечивает «площадку для тестирования до публикации» предлагаемых определений и терминов. К счастью, она состоит не только из одних специалистов. В нее входят представители различных областей, поскольку они в состоянии одновременно вести диалог с отраслевыми экспертами и ставить себя на место «неспециалистов». Способность членов Комиссии быть любопытными и любознательными, следовательно, является решающей для успеха в работе.

Комиссия, которую в настоящий момент возглавляет член Французской академии, писатель и журналист Фредерик Виту, состоит одновременно из обязательных членов и избираемых каждые четыре года необязательных. Обязательными членами являются руководитель Национального управления по продвижению французского языка и языков Франции, который координирует работу сети, Постоянный секретарь Французской академии, специалист по истории России Элен Каррер д'Анкосс, которую представляет ее заместитель, Постоянный секретарь Академии наук (в настоящий момент известный биолог), председатель Французской ассоциации по стандартизации (AFNOR), которого представляет руководитель службы переводов ассоциации, представитель Международной организации франкофонии (OIF), председатель Высшего совета телерадиовещания. Одиннадцать необязательных членов обладают разнообразными компетенциями: генеральный адвокат Кассационного суда, посол, бывшая редактор-корректор газеты Le Monde, редактор научной прессы, генеральный инспектор в области физики и химии, генеральный инженер шахт, дипломат (женщина), профессор по биологии (женщина), два профессора по филологии и одна писательница. Работа постоянного секретариата Комиссии обеспечивается Национальным управлением по продвижению французского языка и языков Франции (DGLFLF).

\section{Роль Комиссии}

Комиссия по обогащению французского языка играет роль посредника между профессионалами и широкой аудиторией. 
- en consultant simultanément les partenaires du dispositif (par exemple l'Office québécois de la langue française ou l'Association française de normalisation) pour recueillir leur avis,

- ou encore, en faisant ponctuellement appel à des spécialistes extérieurs au réseau, notamment lorsque la notion étudiée relève d'une branche du domaine d'étude que les experts ne maîtrisent pas suffisamment.

Puis le terme est instruit dans l'intervalle des réunions par un ou plusieurs rapporteurs chargés de présenter la notion aux autres experts, d'ébaucher la définition et de faire des propositions de terme. La rédaction définitive de la définition ainsi que celle des éventuelles notes et le choix du terme se font en réunion. Ce sont les experts qui rédigent, les linguistes présents ne sont là que pour les conseiller et leur transmettre leur savoir-faire terminologique. Ainsi, au fil des réunions et de l'étude des termes les experts acquièrent eux-mêmes une expertise terminologique et deviennent de plus en plus autonomes.

Lorsqu'un groupe d'experts dispose d'une série aboutie de termes, il la transmet à la Commission d'enrichissement de la langue française.

\section{La Commission d'enrichissement de la langue française = le « banc d'essai »}

La Commission, actuellement présidée par le romancier et journaliste Frédéric Vitoux, membre de l'Académie française, est composée à la fois de membres de droit et de personnalités renouvelées tous les quatre ans. Les membres de droit sont le Délégué général à la langue française et aux langues de France, qui coordonne le réseau, le Secrétaire perpétuel de l'Académie française (l'historienne russophile Hélène Carrère d'Encausse) représenté par son Directeur de Cabinet, le Secrétaire perpétuel de l'Académie des sciences (actuellement une biologiste renommée), le président de l'Association française de normalisation (AFNOR), représenté par le responsable du service de traduction de l'organisme, le représentant de l'Organisation internationale de la Francophonie (OIF), le président du Conseil supérieur de l'audiovisuel (CSA). Les onze personnalités ont des compétences variées : une avocate générale de la Cour de cassation, un ambassadeur, une ancienne correctrice-réviseuse du journal Le Monde, un éditeur de presse scientifique, une inspectrice générale de physique-chimie, un ingénieur général des mines, une diplomate, une professeure de biologie, deux professeurs de lettres, une romancière.

Le secrétariat permanent de la Commission est assuré par la Délégation générale à la langue française et aux langues de France (DGLFLF).

\section{Le rôle de la Commission}

La Commission d'enrichissement de la langue française fait la jonction entre les professionnels et le grand public. Elle veille donc à proposer des définitions et des termes à la fois satisfaisants pour les spécialistes et accessibles au grand public, en premier lieu les agents de l'État, puisque les termes et expressions publiés au Journal officiel sont obligatoirement utilisés à la place des termes et expressions équivalents en langues étrangères a) dans les décrets, arrêtés, circulaires, instructions et directives des ministres, dans les correspondances et documents, de quelque nature qu'ils soient, qui émanent des services et 
Таким образом, она следит за тем, чтобы предложенные определения и термины одновременно удовлетворяли специалистов и были понятны широкой аудитории, в первую очередь государственным служащим, поскольку публикуемые в Официальном бюллетене термины и выражения обязательны к использованию вместо соответствующих обозначений на иностранных языках: а) во всех декретах, постановлениях, циркулярах, инструкциях и директивах министерств, корреспонденциях и документах, которые исходят от служб и государственных учреждений; б) в случаях, предусмотренных статьями 5 и 14 закона от 4 августа 1994 г. об использовании французского языка. В статье 5 говорится о том, что "договоры, в которых участниками являются юридическое лицо государственного права или частное лицо, выполняющее государственную задачу, составляются на французском языке». В статье 14 указано, что «использование фабричного, торгового или служебного знака, в котором присутствует какое-либо иностранное выражение или термин, запрещено для юридических лиц государственного права в случаях, когда существует французское выражение или термин стем же значением».

Комиссия по обогащению французского языка следит, в частности, за гармонизацией предлагаемого словаря как внутри сети для всех областей, так и для других франкофонных организаций по вопросам терминологии, образования неологизмов и стандартизации. Диалог является важным элементом, поскольку Комиссия не вносит никаких изменений без согласия экспертов.

Французская академия, в которую Комиссия передает список принятых терминов, может, в свою очередь, сделать предложения и задать вопросы. В таком случае между группами экспертов, Комиссией по обогащению французского языка и Французской академией может начаться обмен мнениями стем, чтобы прийти к консенсусу.
Как только группа экспертов, Комиссия по обогащению французского языка и Французская академия приходят к согласию по определениям и терминам, Комиссия их публикует в Официальном бюллетене, а Национальное управление по продвижению французского языка и языков Франции распространяет их среди широкой аудитории, главным образом через свой сайт www.franceterme.fr. В то же время группы экспертов при соответствующей поддержке сверху обеспечивают их распространение среди специалистов. Термины становятся обязательными к использованию для государственных служащих, при этом ими могут также пользоваться все франкоговорящие люди.

Несколько тысяч научно-технических терминов (от 250 до 350, в зависимости от года) были созданы благодаря механизму обогащения французского языка:

1973: leasing = crédit-bail, лизинг (появился задолго до того, как благодаря развитию этой системы стало возможно обзавестись автомобилем);

1981: database = base de données, база данных (40 лет назад это был совершенно новый термин, теперь он известен всем);

1983: walkman = baladeur, портативный плеер;

2005: head-up display = affichage tête haute, индикатор на лобовом стекле;

2008: токамак = tokamak (установка, необходимая для термоядерного синтеза, заимствование из русского языка!);

2009: Segway = gyropode, сегвей;

2013: hashtag = mot-dièse, хештег, знак решетки;

2017: returnee, returned foreign fighter $=$ revenant, вернувшийся иностранный боевик (для обозначения террористов DAESH/ ИГИЛ, вернувшихся из Сирии).

Следовательно, для гарантирования успеха политики по внедрению новой терминологии очень важно вовлекать в этот процесс специалистов, с тем чтобы они затем использовали новую лексику. 
des établissements publics de l'État, et b) dans les cas prévus aux articles 5 et 14 de la loi du 4 août 1994 relative à l'emploi de la langue française. L'article 5 porte sur " les contrats auxquels une personne morale de droit public ou une personne privée exécutant une mission de service public sont parties sont rédigés en langue française ». L'article 14 indique que « l'emploi d'une marque de fabrique, de commerce ou de service constituée d'une expression ou d'un terme étrangers est interdit aux personnes morales de droit public dès lors qu'il existe une expression ou un terme français de même sens ".

L'Académie française, qui est destinataire de la liste de termes adoptés par la Commission d'enrichissement de la langue française, peut à son tour faire des suggestions ou poser des questions. Il peut y avoir un « aller-retour » entre groupes d'experts, Commission d'enrichissement et Académie française pour parvenir au consensus. Une fois que le groupe d'experts, la Commission et l'Académie française sont d'accord sur les définitions et les termes, la Commission les publie au Journal officiel et la Délégation générale à la langue française et aux langues de France les diffuse auprès du grand public, principalement grâce à son site de l'internet www.franceterme.fr, tandis que les groupes d'experts, avec l'appui de leur ministère de tutelle, assurent la diffusion auprès des professionnels.

Les termes deviennent d'emploi obligatoire pour les agents de l'administration mais tous les francophones peuvent les adopter.

Plusieurs milliers de termes scientifiques et techniques, de 250 à 350 selon les années, ont été produits par le dispositif d'enrichissement de la langue française et certains sont entrés ou en passe d'entrer dans la langue générale :

1973 : leasing = crédit-bail (bien avant que ce système se répande pour avoir une voiture)

1981 : database $=$ base de données (un terme totalement nouveau il y a 40 ans et désormais connu de tous)

$1983:$ Walkman = baladeur

2005: head-up display = affichage tête haute (sur le pare-brise des véhicules)

2008 : токамак = tokamak (dispositif essentiel en fusion thermonucléaire ; un emprunt au russe !)

$2009:$ Segway = gyropode

2013 : hashtag $=$ mot-dièse

2017 : returnee, returned foreign fighter = revenant (pour désigner les terroristes de DAESH / ISIS de retour de Syrie)

Pour garantir le succès de la politique d'aménagement terminologique, il est donc capital d'impliquer les professionnels dans le processus pour qu'ils utilisent ensuite le vocabulaire. 